\title{
Von der Not zur Tugend: 2020 als Turbobooster für neue und kreative Lehrformate
}

\author{
Cornelia Estner, Daniel Schropp und Tatjana Spaeth
}

\section{Zusammenfassung des Beitrags}

Vor dem Jahr 2020 stand die Universität Ulm hinsichtlich der Digitalisierung innerhalb der Hochschullandschaft eher im hinteren Mittelfeld. Umso größer war der Kraftakt, der im Zuge einer schnellen Umstellung auf digitale Lehre zum Sommersemester 2020 aufgewandt werden musste. Neben der technischen Aufrüstung entstand ein umfangreiches Unterstützungsangebot für Lehrende sowie ein flächendeckendes Multiplikator*innennetzwerk. Diese Maßnahmen wurden durch eine Evaluation begleitet (Lehrendenaustausch, Podiumsdiskussion, Rückmeldungen zum Sonderpreis für engagierte Online-Lehre sowie Studierendenbefragung und Lehrevaluationen) mit dem Ziel, positive Entwicklungen nachhaltig zu implementieren. Die Universität Ulm kann ein überaus positives Fazit ziehen und daraus Empfehlungen für eine gelungene Umstellung auf die Online-Lehre geben.

Schlüsselbegriffe: Online-Lehre $\bullet$ Digitalisierung $\bullet$ Asynchrone Lehrformate $\bullet$ Synchrone Lehrformate - Umstellung

\section{Ausgangslage: Wie startete die Universität ULM in das erste Corona-Semester?}

Die Universität Ulm wurde 1967 als medizinisch-naturwissenschaftliche Hochschule gegründet, ihre mehr als 60 Studiengänge haben ein klares Fächerspektrum mit WiMINT-Schwerpunkt. Als kleinere und überschaubare Universität verfügt die Universität Ulm über einen individuellen Charakter als „Universität unter einem Dach“ mit kurzen Wegen und engen Kontakten zwischen Studierenden und Lehrenden. Typisch für das Studium an der Universität Ulm war bis März 2020 eine an individuelle Bedürfnisse angelehnte Betreuung. Die Lehre setzte stark auf den persönlichen Kontakt zwischen Lehrenden und Studierenden in überwiegend kleinen Gruppen. Dieser Anspruch an Qualität und Stellenwert der Lehre wurde in einem Leitbild festgehalten und 2019 vom Senat der Universität Ulm verabschiedet ${ }^{1}$.

Seit dem Wintersemester 2011 verwendet die Universität Ulm die Lernplattform Moodle. Die Nutzungszahlen stiegen zwar in den Folgesemestern konstant, dennoch besaß Ende 2018 nur

\footnotetext{
${ }^{1}$ Das Leitbild ist online verfügbar: www.uni-ulm.de/leitbild-lehre/
} 
etwas mehr als die Hälfte der Lehrveranstaltungen einen zugehörigen Moodle-Kurs. Der Stand der Digitalisierung war im Vergleich zu vielen anderen Hochschulen entsprechend eher gering, als sich zum Sommersemester 2020 innerhalb kürzester Zeit die Notwendigkeit ergab, die Lehre auf ein digitales Format umzustellen.

\section{Die große Herausforderung: Wie gelingt innerhalb kürzester Zeit die Umstellung auf eine mindestens gleichwertige digitale Lehre?}

In den Vorbereitungen auf das Sommersemester 2020 sah sich die Universität Ulm mit der großen Herausforderung konfrontiert, das durch Präsenzlehre und den persönlichen Austausch geprägte universitäre Leben auf ein adäquates digitales Format umzustellen. Hierfür setzte man einerseits auf den Aufbau neuer, insbesondere technischer Dienste sowie andererseits auf die bereits bewährte gute Vernetzung innerhalb der Universität, wie z. B. Mitarbeiter*innen aus bestehenden Strukturen wie Zentrum für Lehrentwicklung, Studierendenberatung, Kommunikations- und Informationszentrum u.v.m., die vor der Pandemie schon in Projekten und Arbeitskreisen miteinander gearbeitet hatten. Konkret prägten drei Ziele die Umstellungsphase:

1. Die Universität Ulm wollte Lehrenden und Studierenden kurzfristig eine funktionierende Infrastruktur zur Verfügung stellen, die qualitativ hochwertige Lehre digital ermöglicht.

2. Die Universität Ulm wollte Lehrende bei der Umstellung auf digitale Lehre unterstützen und zur didaktisch sinnvollen Verwendung der technischen Infrastruktur befähigen.

3. Die Universität Ulm wollte Netzwerke zur gegenseitigen Unterstützung (re-)aktivieren, die für eine schnelle und unkomplizierte Unterstützung sorgen und in denen Kompetenzen und Ideen ausgetauscht werden.

Diese Ziele sowie die Maßnahmen zu deren Umsetzung werden im Folgenden detailliert vorgestellt.

\subsection{Auf-und Ausbau der technischen Infrastruktur für die digitale Lebre}

Neben einer zügigen Aufrüstung von Serverkapazitäten gab die Leitungsebene der Universität Ulm einen klaren Rahmen für die digitale Lehre vor. Sie orientierte sich damit an der Wirtschaft, in der ein Top-Down-Ansatz für die Implementierung von E-Learning als erfolgreiches Prinzip beschrieben wird (vgl. Oeste/Wegener/Leimeister 2014). Eine ausdrückliche Pflicht zur Nutzung der Lernplattform Moodle sollte gewährleisten, dass Studierende verlässlich die relevanten Informationen, Materialien und Aktivitäten zu allen Lehrveranstaltungen finden. Darüber hinaus sollten Lehrveranstaltungen asynchron angeboten werden. Diese Vorgabe berücksichtigte insbesondere die Bandbreitenkapazitäten der Lehrenden und Studierenden im privaten Raum. Für synchrone Kleingruppenarbeiten und zur interaktiven Arbeit mit Studierenden wurde die Videokonferenzlösung BigBlueButton in Moodle integriert. Für Besprechungen außerhalb des Lehrkontextes stand den Mitarbeiter*innen Cisco WebEx zur Verfügung. Im Interesse des Datenschutzes und auch, um einen effektiven Support und eine zuver- 
lässige Struktur für die Studierenden sicherzustellen, riet die Universitätsleitung von der Nutzung von Fremdtools für die Kommunikation und Materialbereitstellung dringend ab. Aktuelle Änderungen und Updates im Zusammenhang mit der digitalen Lehre wurden in der Regel per Mail und mit Verweis auf zentrale Webseiten der Universität übermittelt. Mit diesen sehr klaren und kanalisierten Vorgaben sowie der technischen Aufrüstung war ein wichtiger Grundstein für eine zügige Umstellung der Präsenzlehre in den digitalen Raum gelegt.

\subsection{Umfangreiches Unterstützungsangebot für Lebrende}

Parallel zum Auf- und Ausbau der technischen Infrastruktur erstellten die Mitarbeiter*innen des Zentrums für Lehrentwicklung (ZLE) in Zusammenarbeit mit dem Kommunikationsund Informationszentrum (kiz) sowie dem Kompetenzzentrum eEducation in der Medizin an der Universität Ulm ein umfangreiches Unterstützungsangebot für Lehrende. Wie von Schumacher, Ademmer, Bülter und Kneiphoff (vgl. 2021) resümiert, zeigte sich auch an der Universität Ulm der größte Bedarf in der Kompetenzentwicklung im Bereich der digitalen Lehre, sowohl in den didaktischen Konzepten und Methoden als auch in der Nutzung der zur Verfügung stehenden technischen Infrastruktur. Innerhalb kürzester Zeit wurde deshalb eine zentrale und ständig aktualisierte Webseite „Alternativen zur Präsenzlehre“2 eingerichtet, die nicht nur Ideen zur digitalen Lehrgestaltung enthält, sondern auch Vorschläge zur Anpassung an und Durchführung im digitalen Raum enthält. Zeitgleich wurden alle Fragen von Lehrenden bei der Umstellung auf die Online-Lehre gesammelt und im Rahmen von „FAQs“ zentral beantwortet. Als weitere Ergänzung boten Selbstlern- und Demokurse in Moodle sowie technische Anleitungen die Möglichkeit, den Einsatz der verfügbaren Tools (Moodle, Opencast, BigBlueButton) für die Lehre kennen zu lernen. Darüber hinaus entstand ein umfangreiches Workshop- und Beratungsangebot für Lehrende, welches intensiv auf individuelle Anliegen bei der Umstellung auf die Online-Lehre einging.

\subsection{Aufbau von Netzwerken für die Umstellung auf digitale Lebre}

Neben diesen Angeboten setzte die Universität Ulm auf formelle und informelle Netzwerke, um die beschlossenen Maßnahmen und Empfehlungen für die Umstellung auf digitale Lehre effizient über die gesamte Universität zu verbreiten. Auf Ebene der Universitätsleitung wurde ein Corona-Krisenstab gegründet, dem Vertreter*innen aller wichtigen Einrichtungen und Statusgruppen angehörten, so dass zentrale Informationen zügig weitergegeben und schnell auf Bedarfe und aufkommende Probleme reagiert werden konnte. Medienaffine Personen aus den Fächern meldeten sich freiwillig als Digitalisierungshelfer*innen ${ }^{3}$, um die Kolleg*innen in ihrem Fach bei der Umsetzung didaktisch sinnvoller digitaler Lehre zu unterstützen. Sie wurden durch die lehrstützenden Bereiche im Rahmen von Workshops und Austauschrunden geschult und unterstützten und fungierten als Multiplikator*innen für die fachspezifische Lehrumstellung. Weitere wichtige Inputs steuerten Netzwerke außerhalb der Universität Ulm bei. Bezüglich der digitalen Lehre erfolgte ein Austausch von Handreichungen und Inspiration

\footnotetext{
${ }^{2}$ Link zur Webseite: www.uni-ulm.de/einrichtungen/zentrum-fuer-lehrentwicklung/alternativen-zur-praesenzlehre/

${ }^{3}$ Diese Bezeichnung wurde universitätsintern verwendet.
} 
insbesondere im Hochschulnetzwerk Digitalisierung der Lehre Baden-Württemberg (HNDBW) sowie im Hochschuldidaktik-Zentrum Baden-Württemberg (HDZ).

Inwieweit mit den dargestellten Maßnahmen eine erfolgreiche Umstellung auf Online-Lehre erfolgen konnte, wurde über verschiedene Wege evaluiert. Im Folgenden werden die Informationsquellen für die Evaluation sowie unsere Erkenntnisse daraus vorgestellt.

\section{Evaluation: Wie erfolgreich konnte die Universität ULM ihre Ziele in der Umstel- lung auf digitale Lehre erreichen?}

Innerhalb von nur sechs Wochen gelang es der Universität Ulm durch das außerordentliche Engagement ihrer Mitarbeiter*innen in Fächern, Fakultäten und lehrstützenden Einrichtungen, die für die digitale Lehre notwendige Infrastruktur und die flankierende Unterstützung der Lehrenden zum Start des Sommersemester 2020 aufzubauen. Im Vordergrund stand dabei zunächst eine sehr zeitnahe Umsetzung, die wenig Raum für Anpassungen an individuelle Bedarfe oder für nachhaltige Planungen ließ.

Bereits im Verlauf des Sommersemesters wandte die Universität Ulm jedoch den Blick auf die Fragen der individuellen Bedarfe und der nachhaltigen Planungen. Ziel der Evaluation war es, sowohl formativ Erkenntnisse zur Anpassung und Erweiterung der Angebote zu gewinnen als auch Konsequenzen für die nachhaltige Gestaltung des digitalen Lehrangebots an der Universität Ulm abzuleiten. Im Vordergrund standen hierbei die Ziele, die auch die Umstellungsphase prägten. Die technische Infrastruktur sollte gelingende Online-Lehre ermöglichen, Lehrende sollten bei der Umstellung ihrer Lehre effektiv unterstützt werden und Netzwerke zum Austausch von Lehrkonzepten und -erfahrungen sollten gefördert werden. Das Evaluationskonzept setzte dabei auf verschiedene Informationsquellen, die im Folgenden in ihrer Methodik und den Ergebnissen eingehender beschrieben werden. Erkenntnisse zogen wir aus dem Austausch mit Lehrenden (z. B. in Beratungsgesprächen, Austauschrunden mit Professor*innen und Mittelbau, über Gremienrückmeldungen; s.a. Kapitel 3.1), aus einer Online-Podiumsdiskussion, den Nominierungen für einen Sonderpreis für engagierte Lehre sowie aus der ohnehin regelmäßig stattfindenden Studierendenbefragung. Die universitätsinterne Studierendenbefragung wird einmal pro Jahr unter Einbindung der Fachbereiche und der Studierendenvertretung von der Stabsstelle Qualitätsentwicklung der Universität Ulm durchgeführt. Die Ergebnisse werden im Rahmen des Berichts zur Lehre ausgewertet und stehen auf Studienfachebene den Studienkommissionen sowie dem Senatsausschuss Lehre für die Diskussion und Weiterentwicklung der Lehre zur Verfügung.

\subsection{Erkenntnisse aus dem Austausch mit Lebrenden}

Als erste Informationsquelle für die Beurteilung der Umstellung auf die Onlinelehre diente die anekdotische Evidenz aus dem Austausch mit Lehrenden und insbesondere auch den Digitalisierungshelfer*innen. In Beratungsgesprächen, Austauschrunden für Lehrende im Mittelbau bzw. für Professor*innen („Didactic Tea Times“) sowie über Rückmeldungen aus den universitären Gremien ergaben sich wichtige Erkenntnisse zu Erfahrungen, Veränderungsbedarfen und offenen Fragen. 
Vor allem in den Wochen vor Semesterstart war die Stimmung unter den Lehrenden einerseits durch große Unsicherheiten bezüglich der organisatorischen und technischen Herausforderungen bei der Umstellung auf die Online-Lehre, aber auch durch eine bemerkenswerte Einsatzbereitschaft und gegenseitige Unterstützung geprägt. Erste Unterstützungsangebote wie die Webseiten „Alternativen zur Präsenzlehre“, die FAQs zur Online-Lehre sowie die Beratungsangebote wurden sehr gut angenommen. Dies drückte sich darin aus, dass Lehrende und Digitalisierungshelfer*innen in den Beratungsgesprächen häufig auf die entsprechenden Webseiten referenzierten, teilweise Nachfragen stellten und sich in den Austauschrunden auch gegenseitig auf die Informationen aufmerksam machten. Die Lehrenden waren in den Beratungsgesprächen sowie den Austauschrunden zunächst insbesondere interessiert an konkreten Handlungsempfehlungen zur didaktisch-organisatorischen Umstrukturierung ihrer Lehre. Schnell zeigte sich jedoch auch ein Bedarf am fachspezifischen und übergreifenden Austausch von Ideen und Lösungen.

Wichtige Fragen, die sich mit zunehmenden Erfahrungen mit der Onlinelehre im Verlauf des Online-Sommersemesters bei vielen Lehrenden ergaben, bezogen sich auf die Interaktion mit den Studierenden. In den vorwiegend asynchronen Lehrformaten fehlte vielen Lehrenden die Rückmeldung, wie gut die Lehrangebote von den Studierenden wahrgenommen wurden und wie gut diese damit zurechtkamen. Die Beratungsfragen und Diskussionen in den Netzwerkangeboten wandten sich in der Folge sehr viel mehr didaktischen Fragen zu, insbesondere interessierten sich die Lehrenden für Methoden für Feedback und Aktivierung der Studierenden.

\subsection{Online-Podiumsdiskussion zum digitalen Sommersemester}

Weitere Einblicke lieferte die gegen Ende des Sommersemesters 2020 an der Universität Ulm veranstaltete virtuelle Podiumsdiskussion ${ }^{4}$ unter dem Motto „Chance oder Frust? Das OnlineSemester an der Universität Ulm“. Ziel der Veranstaltung war, Meinungen und Erfahrungen der an der Lehre beteiligten Akteur*innen zu sammeln, um diese für die konkrete Planung des nächsten Semesters zu nutzen. Es diskutierten die Vizepräsidentin für Lehre, der CIO, zwei Lehrende, zwei Studierende, ein Digitalisierungshelfer sowie ein Mitarbeiter der Qualitätssicherung ihre Sichtweisen auf das digitale Sommersemester und ihre Wünsche in Bezug auf das anstehende Wintersemester. Die Diskussion wurde live über Webex Events sowie auf dem YouTube-Kanal der Universität Ulm übertragen. Die bis zu 200 live anwesenden Zuschauer*innen konnten sich mit Fragen im Webex-Chat bzw. in den YouTube-Kommentaren in die Diskussion einbringen.

In der Podiumsdiskussion beurteilten die Diskutant*innen die Maßnahmen für die Umstellung auf digitale Lehre überwiegend positiv. Die Zufriedenheit mit der technischen Infrastruktur war groß, alle Beteiligten erkannten die große Leistung auf Seiten der lehrstützenden Einrichtungen beim Aufbau der Technik und der begleitenden Unterstützung an, die ein schnelles Erlernen ermöglichte.

\footnotetext{
${ }^{4}$ Eine Aufzeichnung der Veranstaltung sowie eine Zusammenfassung sind auf dieser Webseite verfügbar: kurzelinks.de/podium-sose2020-uulm
} 
Als wichtige Voraussetzung dafür, dass die Umstellung auf die digitale Lehre gelingen konnte, identifizierten die Diskussionsteilnehmer*innen die Unterstützung durch die Digitalisierungshelfer*innen, die als Ansprechpersonen sowohl technisch und didaktisch kompetent beraten als auch die Fachperspektive berücksichtigen konnten. Darüber hinaus stellten diese einen Kommunikationsfluss zwischen den Fachbereichen und zu den lehrstützenden Einrichtungen sicher. So äußerte ein an der Podiumsdiskussion beteiligter Digitalisierungshelfer: „Die Lösung, die mit den Digitalisierungshelfern entwickelt wurde, war unglaublich durchschlagend [...] weil plötzlich alle, die mit Digitalisierung beschäftigt waren in unterschiedlichen Fachbereichen miteinander kommuniziert haben."

Die Anstrengungsbereitschaft und das Engagement machten sich jedoch auch in einer überaus großen Arbeitsbelastung sowohl der Lehrenden, die viel Zeit und Aufwand in die Produktion asynchroner Lehrmaterialien investierten als auch der Studierenden bemerkbar. Ein Mitarbeiter der Qualitätssicherung berichtete in der Diskussion aktuelle Ergebnisse aus der Lehrevaluation: „Wir haben uns auch den Workload mal angeschaut. [...] Wenn man sich das mal [...] über alle Fächer hinweg anschaut, dann haben wir im Durchschnitt etwa eine Stunde mehr im Vergleich zu den letzten Semestern. "Der Mehraufwand auf Seiten der Studierenden war nach Auffassung der Diskutant*innen allerdings auch ein Resultat einer intensiveren inhaltlichen Auseinandersetzung, da die Studierenden mehr Lernzeit aufbrachten. Ein Lehrender äußerte so auch: „Ich habe den Eindruck, dass die Studierenden angefangen haben, sehr viel intensiver plötzlich zu arbeiten."

Mit Blick auf das folgende Wintersemester 2020/21 war das Resümee der Podiumsdiskussion teilweise auch von Frustration geprägt. Gerade die sozialen Aspekte in der Hochschullehre, sowohl der Austausch und Feedback zwischen Lehrenden und Studierenden als auch die Bildung von Unterstützungsstrukturen und sozialen Beziehungen zwischen den Studierenden waren durch die Onlinelehre deutlich erschwert. Insbesondere für die Studienanfänger*innen wünschten sich die Diskutant*innen Präsenzveranstaltungen.

Zusammenfassend kann aus der Podiumsdiskussion das Fazit gezogen werden, dass die Ziele der Universität Ulm in Bezug auf die Umstellung auf die Onlinelehre weitestgehend erfüllt wurden: Die technische Infrastruktur und die Unterstützungsmaßnahmen für die Lehre wurden von den Diskutant ${ }^{*}$ innen positiv beurteilt. Sie stellten auch fest, dass sich tragfähige Netzwerke etabliert hatten, in denen ein Austausch über (online-basierte) Lehre stattfindet. Jedoch fehlten vielen Lehrenden noch passende Ideen für eine funktionierende Kommunikation und Feedbackkultur mit den Studierenden.

\subsection{Sonderpreis für engagierte Lebre im Online-Sommersemester}

Als Anerkennung für die Anstrengungen der Lehrenden und um Erkenntnisse zu gewinnen, welche Merkmale der Onlinelehre für Studierende entscheidende Erfolgsfaktoren waren, lobte das Zentrum für Lehrentwicklung einen „Sonderpreis für engagierte Lehre im Online-Sommersemester"s aus. Studierende konnten Lehrende über ein Online-Formular für den Preis

\footnotetext{
${ }^{5}$ Siehe auch: kurzelinks.de/zle-sonderpreis-sose2020 für eine ausführliche Darstellung sowie für die Liste der Preisträger*innen
} 
nominieren, die Nominierung sollte durch ein aussagekräftiges Statement begründet werden. Diese Statements wurden allen nominierten Lehrenden zurückgespiegelt und hinsichtlich der Qualitätsmerkmale der Onlinelehre aus Sicht der Studierenden ausgewertet.

An der Universität Ulm waren im Sommersemester 2020 insgesamt 9.598 Studierende eingeschrieben, die Lehrveranstaltungen wurden von ca. 2.300 Lehrenden angeboten (davon 227 Professor*innen). Insgesamt gingen 831 Nominierungen für 189 Lehrende über das OnlineFormular ein, dies entspricht durchschnittlich 4,4 Nominierungen pro Person (Range: $1-51$ Nominierungen/Person). Die Nominierungen verteilten sich relativ gleichmäßig über die vier Fakultäten der Universität Ulm. Die Statements zur Begründung waren im Durchschnitt 252,19 Zeichen lang $(S D=251,80$, max. 4.173).

In der Auswertung der Nominierungsstatements zeigte sich besonders häufig, dass Studierenden insbesondere eine zuverlässige und zielführende Betreuung wichtig war. Nahezu zwei Drittel der Statements (65\%) enthielten Aussagen dazu, dass die nominierten Lehrenden Wege etablierten, um auf Fragen und Rückmeldungen der Studierenden einzugehen (z. B.: „Er stand jede Woche für Fragen über BBB zur Verfügung. “) und die Studierenden zum Lernen zu motivieren (z. B. „Ein sympathischer, vorbildlicher Dozent, der seine Aufgabe und Verantwortung im Bereich Lehre ernst nimmt und dem der Erfolg der Studierenden am Herzen liegt."). Die Methoden und Ideen waren dabei vielfältig, z. B. wurden Sprechstunden über BigBlueButton, per Chat oder auch telefonisch angeboten oder Fragen in Diskussionsforen oder per E-Mail beantwortet.

Die zweithäufigste Begründung, die sich in den Nominierungs-Statements fand, bezog sich auf die Materialien, die die Lehrenden zum Lernen zur Verfügung stellten (42\%). Besonders oft wurden hier die mit viel Aufwand von den Lehrenden produzierten Videos genannt (z. B.: „In seinen Videos hat er sehr gut die wichtigsten Punkte erklärt.“). Auch Skripte wurden häufig als hilfreiche Lernressource erwähnt oder auch die Kombination verschiedener Lernmaterialien („Die ausführlichen und zahlreichen Erklärvideos waren qualitativ so gut, dass mir nichts gefehlt hat im Vergleich zu einem ,normalen' Semester mit Präsenzvorlesungen. Das erweiterte Skript und die Folien der Videos haben es sehr erleichtert, die Vorlesungsinhalte später zu wiederholen.“).

In $37 \%$ ihrer Statements begründeten die Studierenden die Nominierungen auch damit, dass sie bei ihren Lehrenden eine große Motivation und den herausragenden Aufwand für die Lehre wahrnahmen und honorieren wollten. Dies kam z. B. in Aussagen wie: „Man hat gemerkt, dass in die Umstellung auf Online-Lehre sehr viel Mühe und Arbeit gesteckt wurde“ zum Ausdruck.

Etwa ein Fünftel (21\%) der Statements lobte auch die gute Struktur der Lehrveranstaltungen. Studierende empfanden die übersichtliche Aufbereitung der Lerninhalte, -materialien und -aktivitäten als besonders hilfreich für ihr Selbststudium. So stand beispielsweise in einem Statement, die nominierte Lehrperson habe ,jede Woche einen guten Zeitplan uns Studierenden an die Hand gegeben damit jeder die Möglichkeit bekam, genau nachzuvollziehen wie viel Lerninhalt in einer bestimmten Zeit erlernt werden sollte." 
Mit $14 \%$ nur selten zur Begründung herangezogen wurden Aktivitäten, mit denen die Lehrenden die Selbstlernphasen unterstützten (z. B. „man wurde durch die Quizfunktion motiviert sich aktiv mit den Inhalten auseinanderzusetzen."). Unklar ist, ob dies daran liegt, dass Aktivitäten wie Selbsttests, Fallbeispiele oder Übungsaufgaben nur selten eingesetzt wurden oder ob Studierende diese als weniger hilfreich für ihren Lernprozess wahrnahmen. Aus der Podiumsdiskussion und dem Austausch mit den Akteur*innen in der Lehre hatte sich bereits gezeigt, dass einerseits Studierende durch die Online-Lehre einen erhöhten Workload hatten, andererseits aber auch Lehrende berichteten, dass solche Angebote nur von einer Minderheit der Studierenden wahrgenommen würden.

Alle nominierten Lehrenden erhielten die Statements zu ihrer Person per E-Mail. Ausgezeichnet mit dem Sonderpreis für engagierte Lehre im Sommersemester 2020 wurden die drei Lehrenden, die insgesamt am häufigsten nominiert wurden (36, 38 und 51 Nominierungen) sowie die Lehrenden, die in den vier Fakultäten jeweils am häufigsten von den Studierenden für den Sonderpreis vorgeschlagen wurden (19, 23, 27 und 33 Nominierungen). Weiterhin wurden fünf Lehrende nach Auswertung der Statements für ihre Leistungen ausgezeichnet: (1) der am häufigsten nominierte studentische Lehrende, (2) ein Lehrender, bei dem aus den Statements hervorging, dass er die Lehre gemeinsam mit den Studierenden weiterentwickelte, (3) ein Lehrender, der die soziale Eingebundenheit der Studierenden im Besonderen förderte, (4) ein Lehrender, der seine Lehre besonders unterhaltsam und unterstützend gestaltete und (5) ein Lehrender, der sich regelmäßig Feedback zum Workload und Verständnis der Studierenden einholte.

Die Ergebnisse der Auswertung des Sonderpreises für engagierte Lehre im Sommersemester 2020 fassten wir auf einer Webseite zusammen, sie flossen zudem in Beratungsgespräche und Workshops für Lehrende ein. Auch wurden sie in Austauschrunden mit den Lehrenden diskutiert. Lehrende meldeten zudem zurück, dass sie es als sehr motivierend empfunden hatten, über die Nominierungen zum Sonderpreis für engagierte Lehre Feedback zu ihrer Lehre erhalten zu haben.

\subsection{Studierendenbefragung und Lebrevaluationen}

In der jährlich im Sommersemester stattfindenden universitätsweiten Studierendenbefragung bewerteten rund $70 \%$ der Befragten den Umgang der Universität Ulm mit den Herausforderungen der COVID-19-Pandemie mit „gut“ bis „sehr gut“. Als besonders hilfreiche OnlineLehrmittel nannten die Studierenden die von der Universität bereitgestellten Tools Moodle (83\%), Opencast (72\%) und BigBlueButton (64\%). Dies zeigt, dass das Ziel, zentrale Ressourcen für die Distribution von Lehrmaterialien und den Austausch mit Studierenden zur Verfügung zu stellen, gelungen ist.

Viele Studierende konnten der Aussage, dass die Kommunikation mit den Lehrenden problemlos verlief, zustimmen (55\%). Als verbesserungswürdig wurde jedoch das Feedback von Lehrenden in der Online-Lehre beurteilt. Die Aussage „Ich hätte mir in der Online-Lehre mehr Feedback von den Lehrenden gewünscht“ bewerteten $44 \%$ der Befragten mit „trifft voll und ganz zu“ (14\%) bzw. „trifft eher zu“(30\%). Auch die Gelegenheit zu sozialen Kontakten 
kam laut den Ergebnissen der Studierendenbefragung eher zu kurz: Die Mehrheit der Studierenden stimmte der Aussage „Auch während der Online-Lehre gab es genug Gelegenheit zur Kommunikation und Interaktion mit Kommilitonen " überhaupt nicht (26\%) bzw. eher nicht $\mathrm{zu}(34 \%)$. So verwundert es auch nicht, dass sich in den offenen Verbesserungsvorschlägen häufig der Wunsch nach einer besseren Kommunikation und Interaktion fand.

\section{Lessons Learned: Was können andere Hochschulen aus dem Umstellungsprozess der Universität Ulm lernen?}

Basierend auf den Erfahrungen bei der Umstellung auf die Online-Lehre an der Universität Ulm scheinen folgende Gegebenheiten für eine gelungene Umsetzung notwendig zu sein.

\subsection{Rabmenbedingungen}

Die Basis für die erfolgreiche Umstellung auf digitale Lehre war an der Universität Ulm sicherlich die Bereitstellung einer funktionierenden technischen Infrastruktur. Aber auch die Vorgabe eines konkreten, klar abgesteckten Rahmens zur technischen Organisation der Lehre erwies sich als sinnvoll, um Verlässlichkeit für Lehrende im Support und für die Auffindbarkeit der Lehrressourcen auf Seiten der Studierenden zu schaffen.

\subsection{Gestaltungshinweise für die Online-Lehre}

Grundsätzlich existieren vielfältige Gestaltungshinweise für die Online-Lehre (z. B. Arnold et al. 2018; Blessing/Harst 2011; Ebner/Schön 2013). Im Zuge der zwingend notwendigen Digitalisierung haben sich aus unserer Sicht einige funktionierende Minimalanforderungen herauskristallisiert, die sich als überaus erfolgreich für die Online-Lehre bewährt haben und auch künftig in jedem Fall berücksichtigt werden sollten: Zunächst sollte in der Qualifizierung der Lehrenden eine stabile Grundlage (z. B. Grundkenntnisse in der Hochschuldidaktik kennen, grundlegende Funktionen der Lernplattform nutzen können, einfache Aufzeichnungsmöglichkeiten von Vorlesungen kennen) geschaffen werden, um darauf aufbauend dann fachspezifische und individuelle Angebote zu machen. Darüber hinaus muss dem Thema der Kommunikation und Interaktion besondere Aufmerksamkeit gewidmet werden.

In der Evaluation zur Umstellung auf die Online-Lehre stellte sich die Interaktion zwischen Lehrenden und Studierenden als besonders kontrovers heraus. Einerseits beklagten sowohl Studierende als auch Lehrende, dass sie nicht ausreichend Feedback von der jeweilig anderen Gruppe erhielt. Die gelingende Kommunikation war für Studierende so auch der wichtigste Grund, Lehrende für den Sonderpreis für engagierte Lehre zu nominieren. Die Realisierung von Flipped Classroom-Ansätzen (vgl. z. B. Kenner/Jahn 2016) scheint für beide Seiten hier besonders erfolgversprechend zu sein: Etablieren die Lehrenden die Möglichkeit zum synchronen Austausch per Videokonferenz, in dem nicht nur Fragen gestellt werden konnten, sondern auch Inhalte aufbauend auf Selbstlernphasen vertieft werden, erhalten sowohl sie als auch die Studierenden die Möglichkeit zum fachlich basierten Austausch und Rückmeldung über die Lernfortschritte und Verstehensprozesse.

Basierend auf den Rückmeldungen der Studierenden und Lehrenden empfiehlt es sich bei der Gestaltung der synchronen Lehre im digitalen Raum, wenn diese Zeit sehr intensiv zum 
Austausch und zur Interaktion genutzt wird. Didaktisch kann dies einerseits durch die Kleingruppenarbeit in sogenannten Breakout-Rooms erfolgen, die eine hohe Aktivität und Interaktion untereinander auf Studierendenseite erfordern. Andererseits bietet es sich an, die Aufmerksamkeit mit aktivierenden Tools (z. B. Abfragetools wie mentimeter, Kahoot, usw. oder systemintegrierte Abstimmungstools) zu steigern (vgl. Pianos/Burblies/Krüger 2018).

Auch für asynchrone Arbeitsphasen erwies es sich als sinnvoll, Lehrende auf Aktivierungsmethoden hinzuweisen (siehe z. B. Entner/Fleischmann/Strasser 2021). Im asynchronen Bereich sehen wir zudem die Relevanz, Studierenden klare Strukturen zu präsentieren. Kleinschrittige (z. B. wochenweise) Deadlines können Studierende dabei unterstützen, ihren Lernstoff zu priorisieren und ihre Zeiteinteilung besser zu gestalten. Gleichzeitig zeigte sich an der Universität Ulm, dass Lehrende auch dafür sensibilisiert werden müssen, den Workload der Studierenden zu berücksichtigen und sie nicht mit zu vielen Aktivitäten zu überlasten.

\subsection{Bestehende Kompetenzen zu Netzwerken bündeln}

Alle beschriebenen Maßnahmen wären nicht möglich gewesen ohne die hervorragende Arbeit der Netzwerke und Arbeitskreise. Über diese konnten die in regelmäßigen und engmaschigen Treffen verabschiedeten wichtigsten und aktuellsten Informationen zuverlässig und schnell an der Universität verbreitet werden. Als zentrale Unterstützung erwiesen sich hierbei die Digitalisierungshelferinnen und -helfer, die ihre Fachkolleg*innen bei der Umstellung auf OnlineLehre unterstützten und für Fragen zur Verfügung standen. Gerade in Hinblick auf den hohen Workload der Lehrenden erscheint es wünschenswert, Netzwerke in der Lehre auch hochschulübergreifend noch weiter zu fördern. So können sich durch die Nutzung und Veröffentlichung von Open Educational Resources z. B. Synergieeffekte ergeben, die einerseits Lehrende bei der Entwicklung und Weiterentwicklung von Lehrmaterialien entlasten und andererseits die Qualität zu steigern und zu einem kreativen Ideenaustausch beizutragen (vgl. Menkor/Spaeth 2021).

Das Fazit an der Universität Ulm in Bezug auf den Corona-,,Turbobooster" für die digitale Lehre ist abschließend durchaus optimistisch und positiv. Im Gedächtnis bleiben wird den Lehrenden und Studierenden sicherlich, wie durch die gemeinsame Anstrengung die Weiterentwicklung der Lehre möglich ist. Auch wenn Präsenzlehre in Hörsälen und Seminarräumen an der Universität wieder uneingeschränkt möglich ist, werden die digitalen Semester mit Sicherheit nachwirken: In einer größeren Wertschätzung des direkten Kontakts und der Interaktion einerseits und andererseits in der didaktisch sinnvollen Nutzung der Freiräume und Vorteile, die sich auch durch die digitale Lehre für das Lernen und Lehren an der Universität ergeben.

\section{Literatur}

Arnold, Patricia/Kilian, Lars/Thillosen, Anne/Zimmer, Gerhard M. (2018): Handbuch E-Learning. Lehren und Lernen mit digitalen Medien. Bielefeld: Bertelsmann.

Blessing, Axel M./Harst, Wolfgang (2011): Vom Leuchtturmprojekt zum Normalfall: Entwicklungstendenzen im Bereich E-Learning an den Pädagogischen Hochschulen. In: Ludwigsburger Beiträge zur Medienpädagogik, 14, 1-8. URL: doi.org/10.21240/lbzm/14/02. 
Ebner, Martin/Schön, Sandra. (2013): L3T - Lehrbuch für Lernen und Lehren mit Technologien. URL: 13t.tugraz.at/index.php/LehrbuchEbner10/issue/view/9/showToc (18.06.2021).

Entner, Cornelia/Fleischmann, Andreas/Strasser, Alexandra (2021): Hochschullehre im digitalen Wandel. Überlegungen zur didaktischen Gestaltung von Präsenz- und Onlinelehre. In: Berendt, Brigitte/Fleischmann, Andreas/Schaper, Niclas/Szczyrba, Birgit/Wiemer, Matthias/Wildt, Johannes (Hrsg.): Neues Handbuch Hochschullehre, 100. Ausgabe, Artikel A 3.30. Berlin: DUZ Verlagsund Medienhaus $\mathrm{GmbH}$.

Kenner, Alessandra/Jahn, Dirk (2016): Flipped Classroom - Hochschullehre und Tutorien umgedreht gedacht. In: Eßer, Alexandra/Kröpke, Heike/Wittau Heidemarie (Hrsg.): Tutorienarbeit im Diskurs III - Qualifizierung für die Zukunft. Münster: WTM Verlag für wissenschaftliche Texte und Medien, S. 35-58.

Menkor, Michèle/Spaeth, Tatjana (2021): OER an Hochschulen verankern: Erfahrungen aus der Universität Ulm und den Hochschulen in Baden-Württemberg. In: Gabellini, Cinzia/Gallner, Sabrina/Imboden, Franziska/Kuurstra, Maaike/Tremp, Peter (Hrsg.): Lehrentwicklung by Openness - Open Educational Resources im Hochschulkontext. Luzern: Pädagogische Hochschule Luzern, S. 17-22.

Oeste, Sarah/Wegener, Robert/Leimeister, Jan M. (2014): Herausforderungen und Best Practices der ELearning Einführung in Unternehmen. Präsentation auf der Multikonferenz Wirtschaftsinformatik, Paderborn. URL: pubs.wi-kassel.de/wp-content/uploads/2014/05/JML_470.pdf (18.06.2021).

Pianos, Tamara/Burblies, Christine/Krüger, Nicole (2018): Boah ist das langweilig! Aktivierende Methoden für IK-Veranstaltungen. 107. Deutscher Bibliothekartag: offen \& vernetzt. URL: opus4.kobv.de/opus4-bib-info/frontdoor/index/index/docId/3319 (18.06.2021).

Schumacher, Fabian/Ademmer, Tobias/Bülter, Sophie/Kneiphoff, Anika (2021): Hochschulen im Lockdown - Lehren aus dem Sommersemester 2020. Arbeitspapier Nr. 58. Berlin: Hochschulforum Digitalisierung. 


\section{Informationen zu den Autor*innen}

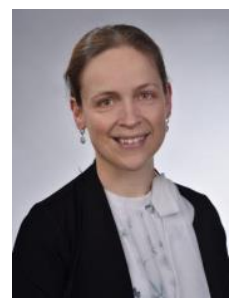

Dr. Cornelia Estner ist Mitarbeiterin im Zentrum für Lehrentwicklung an der Universität Ulm. Sie leitet die Arbeitsstelle für Hochschuldidaktik in Ulm im Rahmen des Hochschuldidaktikzentrums Baden-Württemberg (HDZ).

cornelia.estner@uni-ulm.de

Dr. Daniel Schropp ist an der Universität Ulm Mitarbeiter am Zentrum für Lehrentwicklung und Studiengangskoordinator Lehramt. Teil dieser Tätigkeit ist neben der Weiterentwicklung der Lehramtsstudiengänge die qualitative Verbesserung von Lehrveranstaltungen im WiMINT-Bereich und die Weiterqualifizierung von Hochschullehrenden.

daniel.schropp@uni-ulm.de
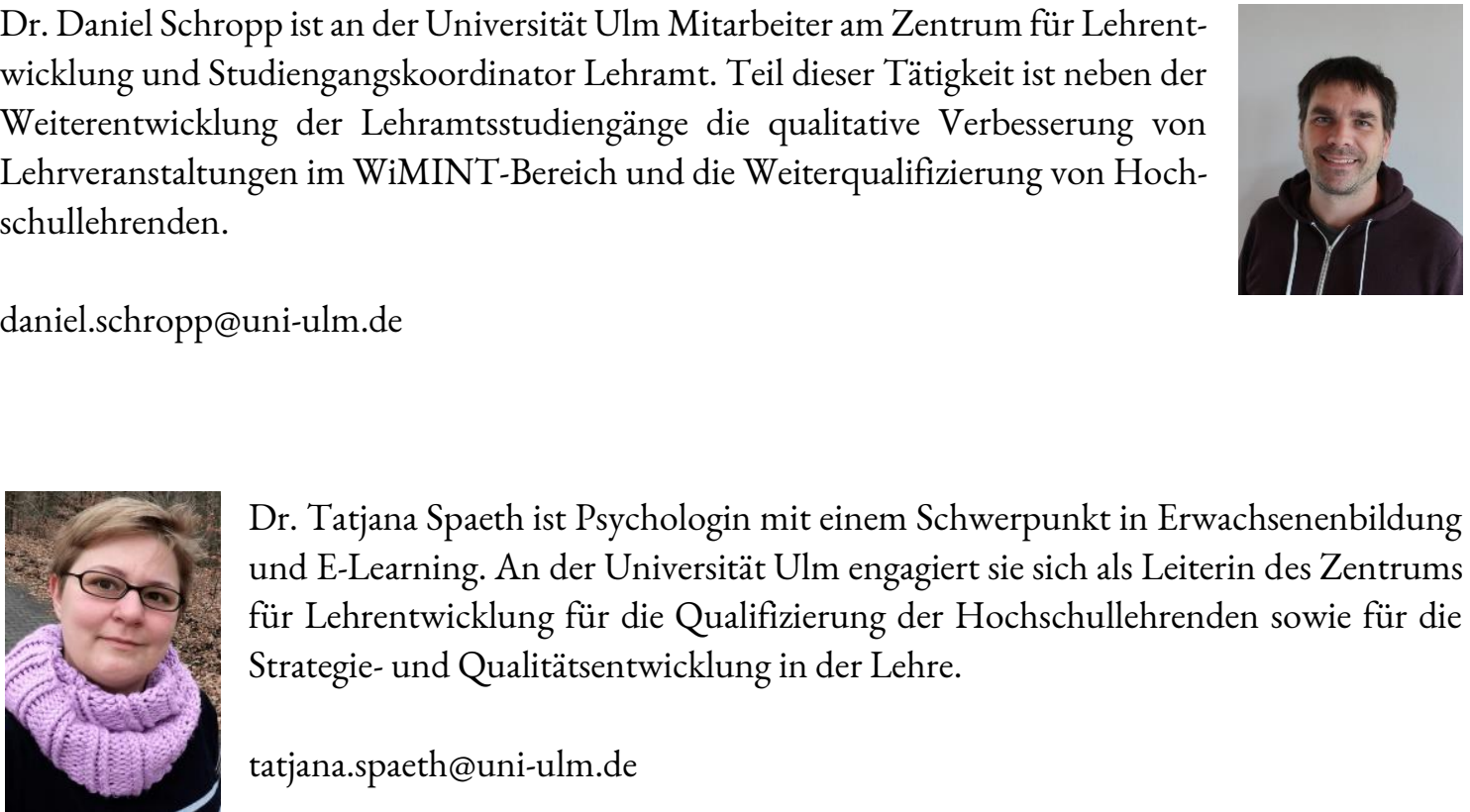

Dr. Tatjana Spaeth ist Psychologin mit einem Schwerpunkt in Erwachsenenbildung und E-Learning. An der Universität Ulm engagiert sie sich als Leiterin des Zentrums für Lehrentwicklung für die Qualifizierung der Hochschullehrenden sowie für die Strategie- und Qualitätsentwicklung in der Lehre.

tatjana.spaeth@uni-ulm.de

\section{Zitationshinweis:}

Estner, Cornelia/Schropp, Daniel/Spaeth, Tatjana (2021): Von der Not zur Tugend: 2020 als Turbobooster für neue und kreative Lehrformate. In: Online-Magazin Ludwigsburger Beiträge zur Medienpädagogik, Ausgabe 21/2021. URL: medienpaed-ludwigsburg.de/ 